\title{
Surgical Removal of Giant Basal Ganglia Arteriovenous Malformations through the Extended Transsylvian Approach Following Preoperative Embolization
}

\author{
-Two Case Reports-
}

\author{
Kazuo Mizol, Takashi Yoshimoto, Yoshihide NAGAMINE*, \\ Akira TAKAHASHI**, and Masayuki EZURA** \\ Department of Neurosurgery, Tohoku University School of Medicine, Sendai; Departments \\ of ${ }^{*}$ Neurosurgery and ${ }^{* *}$ Intravascular Neurosurgery, Kohnan Hospital, Sendai
}

\begin{abstract}
A 12-year-old boy and a 22-year-old male presented with sudden onset of consciousness disturbance with right-sided hemiparesis and aphasia due to massive intracerebral hemorrhage from giant striocapsular arteriovenous malformations (AVMs). Angiography revealed giant AVMs located at the left basal ganglia in both patients. Large frontotemporal craniotomies were performed following staged embolization of the feeding arteries. Through an extended transsylvian approach, the lesions were totally excised with confirmation using intraoperative digital subtraction angiography. The patients showed a transient postoperative worsening of symptoms, but recovered to their preoperative status at discharge. Motor functions of the right upper and lower extremities were graded $4 / 5$ in both patients. These cases demonstrate that combined use of preoperative embolization and intraoperative angiography allows some giant deep AVMs to be resected with encouraging results.
\end{abstract}

Key words: arteriovenous malformations, basal ganglia, transsylvian approach, embolization, intraoperative angiography

\section{Introduction}

Direct surgery for large basal ganglia arteriovenous malformation (AVM) continues to present a difficult problem because of the complex vascular anatomy and the anatomical location adjacent to important neural structures. ${ }^{1,3,16,19)}$ Yasargil ${ }^{21)}$ considered AVMs in this region, particularly the larger lesions, are among the most difficult of all in terms of operability.

We describe two patients with giant basal ganglia (striocapsular) AVMs, which were successfully removed following preoperative staged embolization.

\section{Case Reports}

Case 1: This 12-year-old boy suffered sudden onset

Received January 20, 1995; Accepted March 29, 1995 of consciousness disturbance. Computed tomography $(\mathrm{CT})$ demonstrated a massive intracerebral and intraventricular hemorrhage (Fig. 1). He was in a deep coma. Emergency ventricular drainage using a catheter was performed for the relief of increased intracranial pressure. He progressively recovered from coma following ventricular drainage. A ventriculoperitoneal shunt was then implaced. He was admitted to our hospital under a diagnosis of giant basal ganglia AVM.

He was alert with moderate dysphasia and mild right-sided hemiparesis. His muscle strength was $4 / 5$ in the right upper and lower extremities. Angiography showed the AVM was supplied by many distal branches of the major arteries such as the anterior and posterior choroidal, middle cerebral, anterior cerebral, and posterior cerebral arteries (Fig. 2). This AVM was classified as Grade V according to the Spetzler and Martin system, ${ }^{97}$ as the nidus was $6.5 \mathrm{~cm}$ in size and had deep venous drainage to the galenic system. 


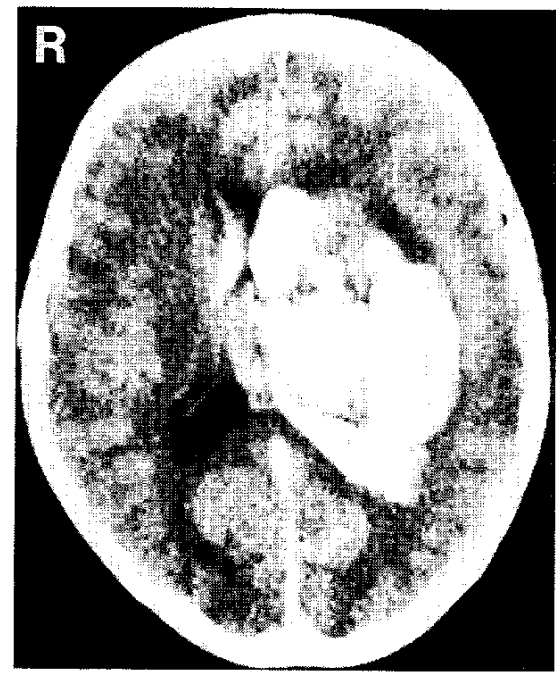

Fig. 1 Case 1. CT scan showing an extensive intracerebral and intraventricular hemorrhage.

Preoperative embolization of this lesion was achieved in four stages. The embolic material was the new liquid estrogen alcohol (EA) and polyvinyl acetate (PVac) solution. ${ }^{12,13)}$ EA has the advantage of obliterating the lesion at the capillary and arteriolar levels due to its chemical inflammatory effects, and PVac polymerizes rapidly upon contact with blood and occludes larger vessels. In some high-flow feeders, 5-0 silk thread or platinum coils were also used. The feeding vessels from the posterior circulation were almost totally obliterated, but those from the anterior circulation were only partially occluded (Fig. 3). He developed no complications following embolization of the malformation. Surgery was undertaken 14 days following the last session of embolization.

After induction of general anesthesia, transfemoral catheterization for intraoperative digital subtraction angiography (DSA) was performed. The left internal carotid and left vertebral arteries were selectively catheterized using two heparinized catheters. These heparinized catheters were left in place during surgery and were flushed continually with heparinized saline. A large frontotemporal craniotomy was performed. The left sylvian fissure was extensively opened, from the carotid cistern to the distal end of the fissure. The superficial sylvian vein bridging between the frontal and temporal lobes was dissected carefully and preserved throughout the surgery. The dilated feeding vessels from the posterior communicating, anterior choroidal, and middle cerebral arteries were clipped.

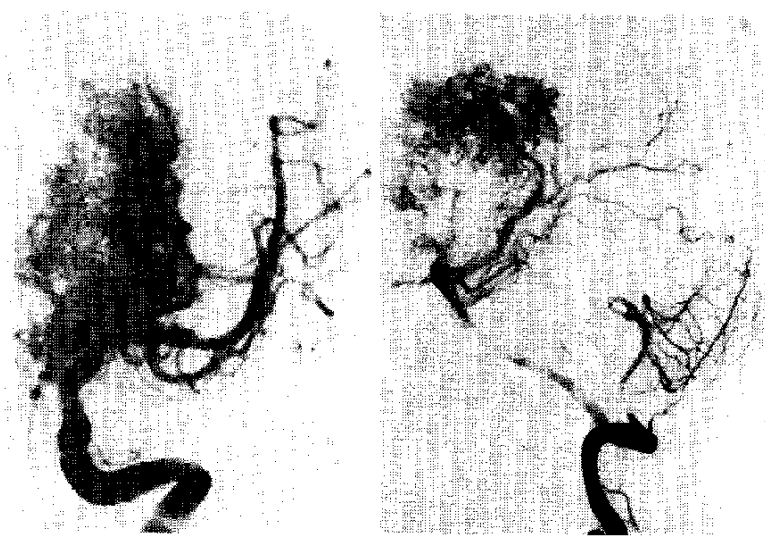

Fig. 2 Case 1. Preoperative angiograms demonstrating a giant AVM in the left basal ganglia. The malformation is supplied by branches of the left internal carotid artery, lenticulostriate arteries, and posterior cerebral artery with venous outflow predominantly to the galenic system. left: anteroposterior view of left internal carotid angiogram, right: lateral view of left vertebral angiogram.

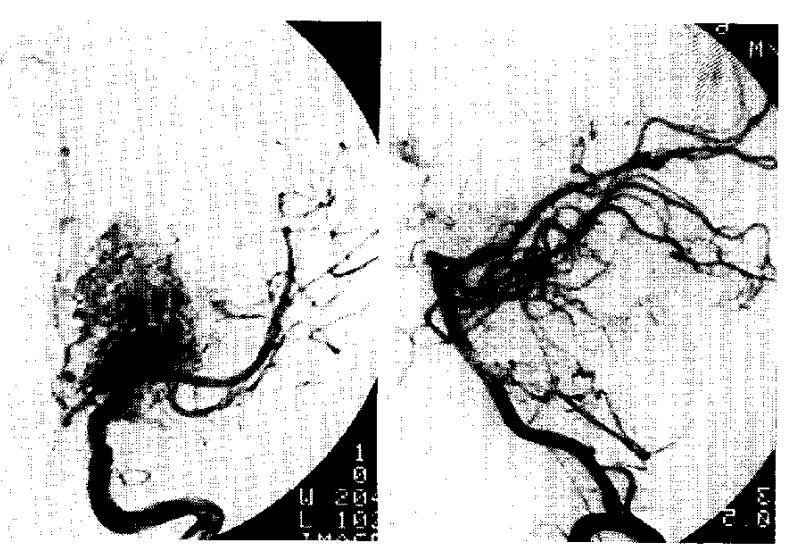

Fig. 3 Case 1. DSAs showing moderate reduction of the AVM size due to preoperative embolization. Angiographic visualization of the feeding vessels from the posterior cerebral artery was markedly reduced. left: anteroposterior view of left carotid angiogram, right: lateral view of left vertebral angiogram.

Incision of the insular cortex exposed a large cavity on the lateral margin of the AVM which was the result of a previous hemorrhage (Fig. 4 left). The wall of this cavity was xanthochromic and provided a good cleavage plane of the malformation. Using bipolar coagulation on the surface of the malformation, a dissection plane was created around the 


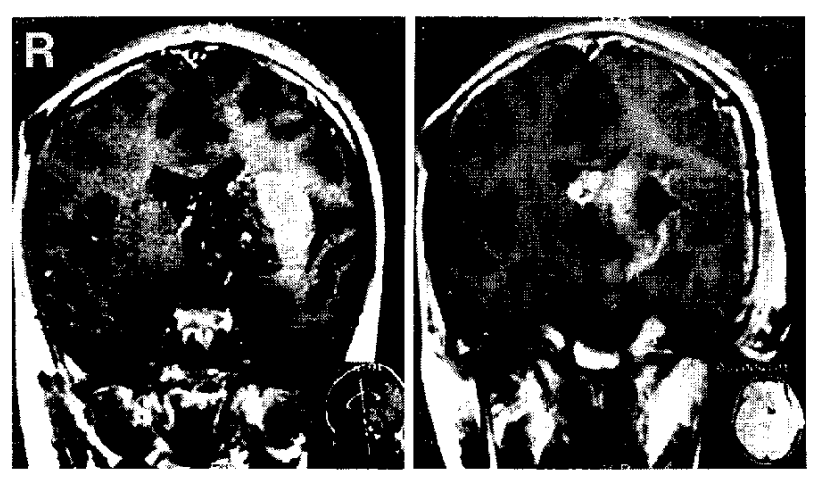

Fig. 4 Case 1. left: Preoperative $T_{1}$-weighted magnetic resonance images demonstrating a giant AVM in the left basal ganglia, primarily in the striocapsular region. The anatomical relationship of the malformation to the internal capsule was difficult to elucidate. Note the large hematoma cavity lateral to the malformation, which facilitated surgical removal of the malformation. right: Postoperative $T_{1}$ weighted magnetic resonance images with gadolinium enhancement, demonstrating that the most medial part of the malformation, which had been completely obliterated by preoperative embolization, was not removed.

malformation. As the dissection advanced deeper to the paraventricular area, we found that the medial segment of the nidus was almost completely obliterated as a result of preoperative embolization. We did not remove the deepest part of the malformation, because intraoperative DSA confirmed the completeness of embolization in this region, which seemed to involve the pyramidal tract (Fig. 4 right). The final intraoperative DSA demonstrated total excision of the malformation (Fig. 5).

He awoke promptly from the operation with a slight worsening of his speech impairment and right hemiparesis ( $2 / 5$ weakness), which recovered gradually. He was discharged 1 month after the operation. His neurological status was essentially the same as when he was first referred to our hospital. Motor examination revealed $4 / 5$ weakness in the right upper and lower extremities. He was able to walk by himself and carry on a meaningful conversation despite a mild dysphasia. He had signs of dyscalculia that had been present preoperatively. Neuroophthalmological examination showed that his right homonymous hemianopsia improved after surgery to become right inferior quadrantanopsia. Postoperative angiography 20 days after the operation demonstrated no residual malformation.

Case 2: This 22-year-old male presented with a sec-

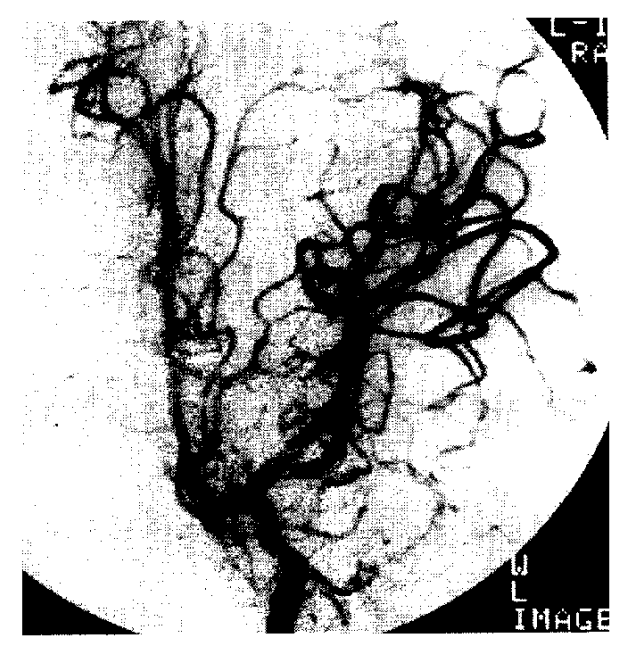

Fig. 5 Case 1. Intraoperative left carotid DSA (anteroposterior view) after resection of the malformation, demonstrating total excision of the malformation.

ond episode of intracerebral and intraventricular hemorrhage. At 19 years of age he had suffered intracranial bleeding and became comatose. CT revealed a massive intraventricular hemorrhage, and angiography revealed a giant AVM, $7 \mathrm{~cm}$ in diameter, in the left basal ganglia. The feeding vessels originated from the left posterior communicating artery, anterior choroidal artery, $\mathrm{A}_{1}$ segment of the anterior cerebral artery, numerous dilated lenticulostriate arteries, insular segments of the middle cerebral artery, and perforating branches of the posterior cerebral artery (Fig. 6). The draining veins from the malformation emptied into the superficial sylvian vein and into the basal vein of Rosenthal. This lesion was considered a Grade V AVM according to Spetzler and Martin. ${ }^{9)}$ Surgical treatment of the AVM was declined and only continuous ventricular drainage was performed. His level of consciousness improved gradually after the ventricular drainage, and he was able to live an almost normal life.

Three years later he had a second major intracranial bleeding. He became comatose again. CT demonstrated a large intracerebral hematoma at the left basal ganglia and intraventricular hemorrhage (Fig. 7 left). He progressively improved, but exhibited persistent right hemiparesis (4/5 weakness), right homonymous hemianopsia, and moderate expressive aphasia. He was referred to our hospital for treatment of this giant striocapsular AVM.

Staged preoperative embolization was done in six sessions using the same liquid embolic materials as in 

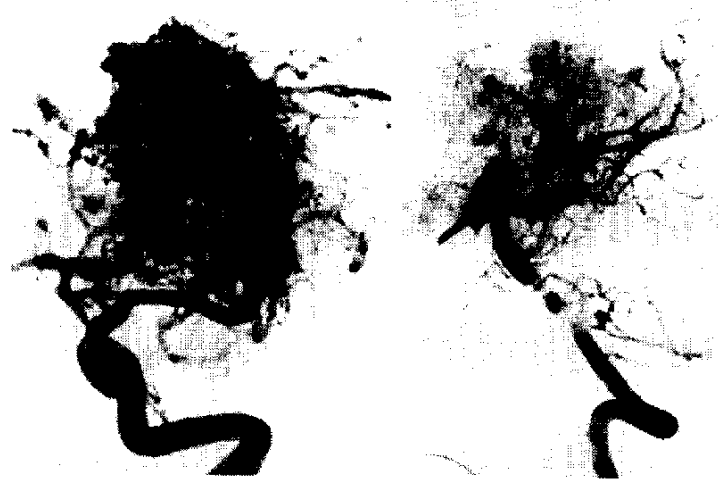

Fig. 6 Case 2. Preoperative angiograms demonstrating a huge AVM in the left basal ganglia. The malformation is supplied by branches of the left internal carotid artery, lenticulostriate arteries, insular segments of middle cerebral artery, and posterior cerebral artery. Venous drainage is primarily into the basal vein of Rosenthal. Note the numerous feeding branches from the middle cerebral artery. left: anteroposterior view of left internal carotid angiogram, right: lateral view of left vertebral angiogram.
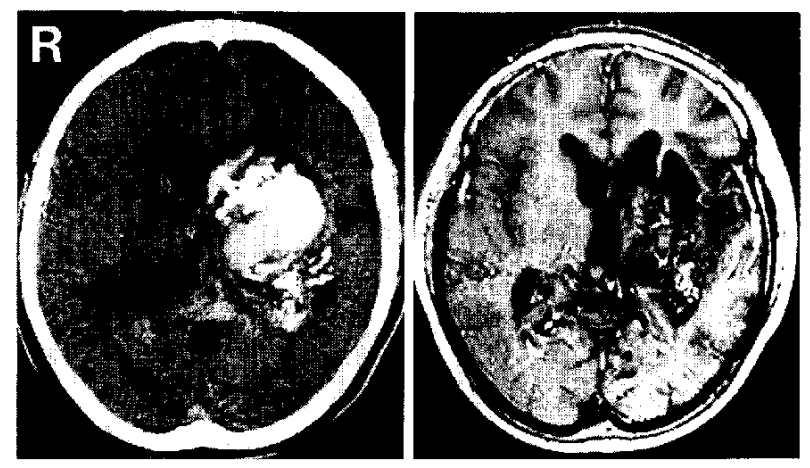

Fig. 7 Case 2. left: CT scan after the second intracranial hemorrhage. right: Preoperative $\mathrm{T}_{1}$-weighted magnetic resonance image demonstrating the hematoma cavity anterior to the malformation. The anatomical relationship between the malformation and the internal capsule was difficult to define.

Case 1. Angiography after the final embolization showed that the AVM was slightly diminished in size (Fig. 8). However, the AVM was considered too large for radiosurgery.

A two-stage resection of the malformation was planned. Occlusion of the main feeding vessels in the

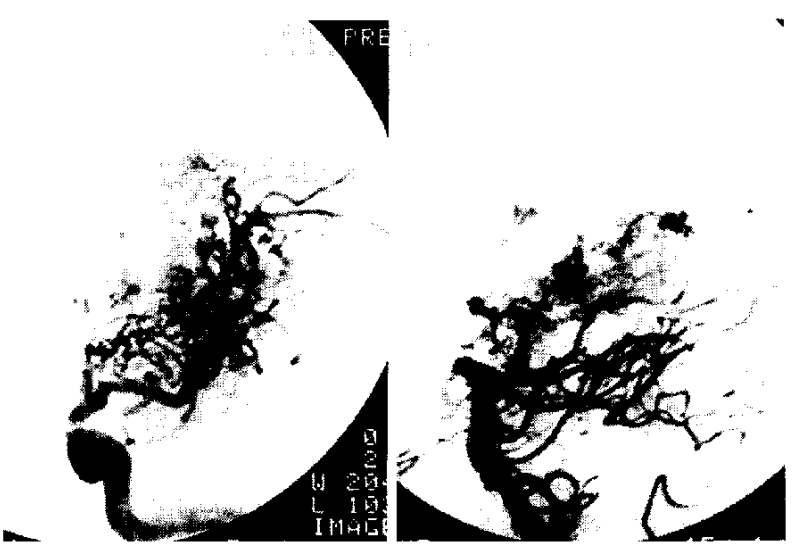

Fig. 8 Case 2. DSAs following the final preoperative embolization, indicating that the AVM was slightly diminished in size as a result of embolization. left: anteroposterior view of left carotid angiogram, right: lateral view of left vertebral angiogram.

first operation and excision of the lesion at the second. The first operation was undertaken 2 weeks following the last session of embolization. Selective transfemoral catheterization was performed for intraoperative DSA. A large frontotemporal bone flap was turned on the left side. The proximal sylvian fissure was opened and many feeding perforating branches from the internal carotid, middle cerebral, and anterior cerebral arteries were identified. These vessels were temporarily clipped with miniclips, then intraoperative DSA confirmed that these vessels were proper feeders of the malformation. The vessels were coagulated and divided. Through a subtemporal approach, the dilated feeding arteries from the $\mathrm{P}_{2}-\mathrm{P}_{3}$ segments of the posterior cerebral artery were also occluded by bipolar coagulation. Final intraoperative DSA demonstrated that the size of the nidus had decreased to about a half. He awoke from the operation with slight left oculomotor paresis which improved gradually and had completely resolved within about 1 month. No other new neurological deficits developed.

Forty days after the first operation, a second operation was performed using the same craniotomy and transfemoral catheterization. The sylvian fissure was opened as far as possible, and the surface of insular cortex was widely exposed. After making a small incision on the insular cortex, an old hematoma cavity was found anterior to the nidus (Fig. 7 right). A plane of dissection was created posteriorly to isolate the nidus from the surrounding neural tissue. However, as the dissection proceeded deeper into the 


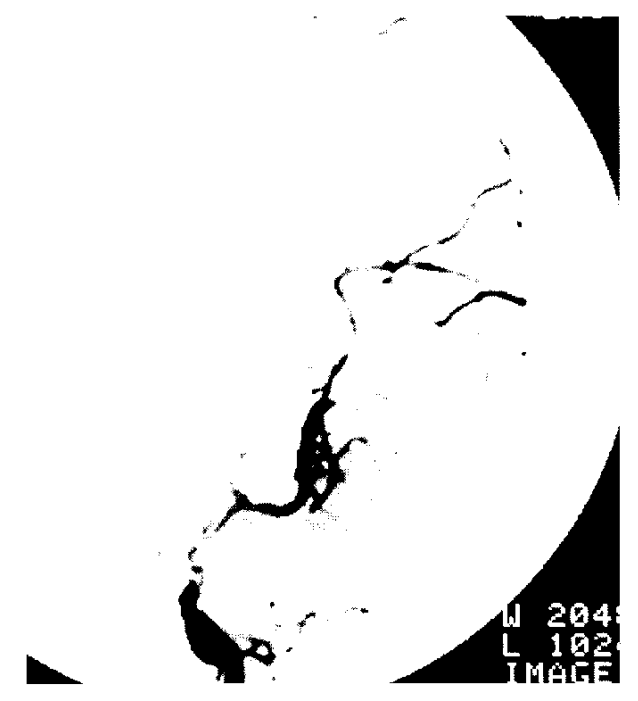

Fig. 9 Case 2. Intraoperative left carotid DSA (anteroposterior view) following resection of the malformation in the second operation, demonstrating total obliteration of the lesion.

basal ganglia, major bleeding occurred from the thin-walled perforating vessels. These small arteries were occluded with miniclips and bipolar coagulation. The main draining vein to the basal vein was preserved until all of the arterial supply to the malformation had been interrupted. The draining vein was then coagulated and divided. Finally, both the anterior horn and the trigone of the lateral ventricle were opened. The thin paraventricular neural tissue along with the third ventricle, which possibly involves the pyramidal tract, was preserved. After apparent total excision of the malformation, intraoperative DSA revealed a small residual AVM on the posterior margin of the resultant cavity. This fragment was located, adjacent to the trigone, and carefully excised. Repeat intraoperative DSA confirmed complete obliteration of the malformation (Fig. 9).

Postoperatively, he showed slight deterioration of right hemiparesis (2/5 weakness). This deficit improved daily and, at discharge about 1 month following the second operation, he could walk by himself with the aid of a stick (4/5 weakness). His motor aphasia which was present prior to surgery improved dramatically after surgery. He was well at follow-up 1 year after the surgery, with improvement in his right hemiparesis. He can walk with a slight limp without any assistance. He now has good gross movements in the right upper extremity but moderate disturbance of fine movements. There is no impairment to speech. His right hemianopsia has remained.

\section{Discussion}

\section{Preoperative embolization}

The recent development of small, highly flexible catheters has greatly increased the use of preoperative embolization in the treatment of AVMs. Preoperative embolization of the AVMs is now routine at many institutions. Extensive embolization of AVMs can diminish the complexity of their vascular supply, making them more accessible to surgical resection, often in a single stage operation. In both of our cases, staged preoperative embolization reduced the number of deep feeding vessels and prevented major bleeding during dissection of the nidus. In Case 1 , an additional subtemporal approach for obliteration of feeding vessels from the posterior cerebral artery was not required, because those vessels had been almost completely interrupted preoperatively. We believe that the surgery in both patients would have involved more risk without preoperative embolization.

The selection of material for embolization is crucial, particularly when resection of an AVM is planned. Many workers have recommended the use of polyvinyl alcohol particles (Ivalon) for preoperative embolization. ${ }^{2,5,10)}$ However, recanalization frequently occurs within a relatively short period after embolization with Ivalon. Preoperative embolization for complex AVMs is usually performed in several sessions. Therefore, both nidus and feeding arteries previously occluded with the Ivalon particles may recanalize during the period before the next step of embolization. We therefore think that Ivalon is not a suitable material for multi-stage preoperative embolization. In contrast, a liquid embolization material has less risk of recanalization compared to Ivalon. However, a conventional liquid material such as a cyanoacrylate glue makes the lesion hard and could make the surgical resection more difficult. ${ }^{6,18)}$ We previously developed a new liquid embolization material, a PVac solution., ${ }^{4,12,13)}$ This is a soft gel and the occluded vessels and the nidus retain their flexibility, so that dissection and retraction of the lesions are quite easy. We recommend PVac solution as the material of choice for staged preoperative embolization.

\section{Surgical approach and intraoperative DSA}

The basic principles for the surgery of a deepseated AVM must take into consideration the safest route to the lesion. Malformations located in the 
anterior thalamus or the head of caudate nucleus are best approached through an interhemispheric transcallosal approach. ${ }^{8,19,20)}$ However, the best approach for removal of the malformations located in the corpus striatum is still unclear. $U^{15)}$ described a transventricular approach through the midfrontal gyrus to excise striocapsular AVMs. This approach would provide a better way to remove the thalamocaudate AVMs located adjacent to the ventricular system. However, there might be more risk of damage to the pyramidal tract for removal of striocapsular AVMs, because the pyramidal tract descends in the white matter between the malformation and the ventricular system.

A transsylvian approach has been described for removing malformations in the region of the insular cortex, lentiform nucleus, or medial temporal lobe. ${ }^{7,11,14,16)}$ We believe that striocapsular malformations are best exposed through an extended transsylvian approach. This approach causes less intraoperative injury to eloquent cortical areas, such as the motor-sensory strip and speech areas. However, in the resection of deep AVMs, the most serious postoperative morbidity would be caused by damage to the pyramidal tract rather than to cortical areas. Accordingly, in the dissection of the medial side of the lesion, preservation of the paraventricular white matter is most important, which possibly involves the internal capsule.

In general, the preoperative study of MR images is useful to define the topographical anatomy of the malformation. However, it is very difficult to predict preoperatively whether large or giant deep-seated AVMs, as in our cases, directly involve the internal capsule, or the nidus is immediately adjacent. We intended to minimize damage to the paraventricular area as much as possible by making the dissection plane as close as possible to the nidus. This presumably resulted in the preservation of motor function in both our patients. However, the more important factor that should be stressed is the use of intraoperative DSA. Intraoperative DSA can confirm that the most critical part of the nidus has been completely embolized, as shown in Case 1, facilitating safer surgical removal to prevent permanent neurological deficit postoperatively. Such confirmation cannot be provided by intraoperative visual inspection alone. We believe that it is not necessary to remove the compartment of the nidus obliterated with our liquid embolic material (EA and PVac), because this region will never recanalize.

The disadvantage of an extended transsylvian approach is the possibility of damage to the superficial sylvian veins. The sacrifice of the veins may cause venous infarction of the frontal lobe in our experience. This will not occur if the veins are widely dissected free from the surrounding arachnoid. The veins should be dissected as extensively as possible using a microdissection technique. We prefer to use the tip of a No. 27 gauge needle as the arachnoid knife for such a sharp dissection.

Our experience with these two patients supports the benefit of combined intravascular and surgical treatment for complex AVM, which until several years ago would have been considered inoperable. ${ }^{17,21)}$ In our cases, the clot cavities formed by previous hemorrhage were apparently helpful for surgery. It is difficult to arrive at a uniform management approach for giant basal ganglia AVMs unless the lesion has previously bled. We consider that asymptomatic patients with giant basal ganglia AVMs do not require aggressive operative treatment.

\section{References}

1) Andreussi L, Cama A, Grossi G, Marino C, Servato R: Microsurgical excision of a strio-insular arteriovenous malformations. Surg Neurol 12: 499502, 1979

2) Andrews BT, Wilson CB: Staged treatment of arteriovenous malformations of the brain. Neurosurgery 21: 314-323, 1987

3) Malik GM, Umansky F, Patel S, Ausman JI: Microsurgical removal of arteriovenous malformations of the basal ganglia. Neurosurgery 23: 209-217, 1988

4) Mizoi K, Takahashi A, Yoshimoto T, Sugawara T, Saito K: Surgical excision of giant cerebellar hemispheric arteriovenous malformations following preoperative embolization. Report of two cases. $J$ Neurosurg 76: 1008-1011, 1992

5) Purdy PD, Samson D, Batjer HH, Risser RC: Preoperative embolization of cerebral arteriovenous malformations with polyvinyl alcohol particles: Experience in 51 adults. $A J N R$ 11: 501-510, 1990

6) Samson D, Ditmore QM, Beyer CWJ: Intravascular use of isobutyl 2-cyanoacrylate: Part 1. Treatment of intracranial arteriovenous malformations. Neurosurgery 8: 43-51, 1981

7) Shi $Y-Q$, Chen $X-C$ : Surgical treatment of arteriovenous malformations of the striatothalamocapsular region. J Neurosurg 66: 352-356, 1987

8) Solomon RA, Stein BM: Interhemispheric approach for the surgical removal of thalamocaudate arteriovenous malformations. $J$ Neurosurg 66: 345351,1987

9) Spetzler RF, Martin NA: A proposed grading system for arteriovenous malformations. $J$ Neurosurg 65 : 476-483, 1986

10) Spetzler RF, Martin NA, Carter LP, Flom RA, Raudzens PA, Wilkinson E: Surgical management of 
large AVM's by staged embolization and operative excision. J Neurosurg 67: 17-28, 1987

11) Sugita K, Takemae T, Kobayashi S: Sylvian fissure arteriovenous malformations. Neurosurgery 21: 714,1987

12) Takahashi A, Suzuki J: Nonsurgical treatment of AVM: Development of new liquid embolization method, in Suzuki $\mathrm{J}(e d)$ : Advances in Surgery for Cerebral Stroke. Tokyo, Springer-Verlag, 1988, pp 215-224

13) Takahashi A, Yoshimoto $T$, Sugawara $T$ : New liquid embolization method for brain arteriovenous malformations: Combined infusion of oestrogen-alcohol and polyvinyl acetate. Neuroradiology 33 (Suppl): 190-192, 1991

14) Taniguchi T, Aoki N, Sasaki T, Mizutani H: “Transinsular approach" for the treatment of a medial temporal arteriovenous malformation. Neurosurgery 32: 863-866, 1993

15) U HS: Microsurgical excision of paraventricular arteriovenous malformations. Neurosurgery 16: 293303,1985

16) U HS, Rosenberg J: Complete recovery from hemianopsia following excision of a giant basal ganglia arteriovenous malformation. Surg Neurol 15: 328330,1981
17) Viale GL, Turtas S, Pau A: Surgical removal of striate arteriovenous malformations. Surg Neurol 14: 321-324, 1980

18) Viñuela FV, Debrun GM, Fox AJ, Girvin JP, Peerless SJ: Dominant hemisphere arteriovenous malformations: Therapeutic embolization with isobutyl-2-cyanoacrylate. $A J N R$ 4: 959-966, 1983

19) Waga $S$, Shimosaka $S$, Kojima $T$ : Arteriovenous malformations of the lateral ventricle. $J$ Neurosurg 63: $185-192,1985$

20) Wilson $\mathrm{CB}$, Martin NA: Deep supratentorial arteriovenous malformations, in Wilson $\mathrm{CB}$, Stein BM (eds): Intracranial Arteriovenous Malformations. Baltimore, Williams \& Wilkins, 1984, pp 184208

21) Yasargil MG: Special surgical consideration of deep central AVMs, in Yasargil MG (ed): Microneurosurgery IIIB. Stuttgart, George Thieme Verlag, 1988, pp 293-316

Address reprint requests to: K. Mizoi, M.D., Department of Neurosurgery, Tohoku University School of Medicine, 1-1 Seiryo-cho, Aoba-ku, Sendai 980-77, Japan. 